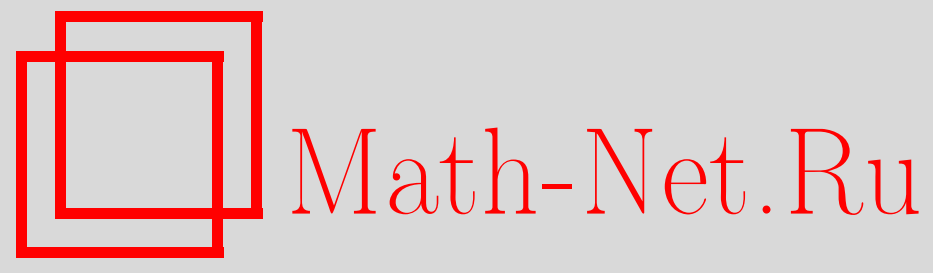

И. В. Семушин, Ю. В. Цыганова, Активная адаптация распределенной мультисенсорной системы фильтрации, Вестн. Сам. гос. техн. ун-та. Сер. Физ.-мат. науки, 2019, номер 4, 724-743

DOI: https://doi.org/10.14498/vsgtu1704

Использование Общероссийского математического портала MathNet.Ru подразумевает, что вы прочитали и согласны с пользовательским соглашением

http://www.mathnet.ru/rus/agreement

Параметры загрузки:

IP : 34.227 .88 .159

26 апреля 2023 г., 13:51:21

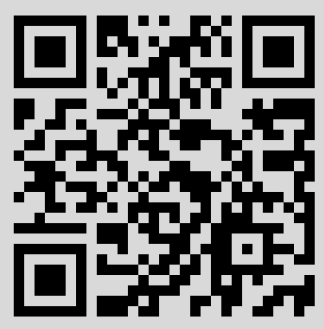


УДК 681.5.015

\title{
Активная адаптация распределенной мультисенсорной системы фильтрации
}

\author{
И. В. Семушин, Ю. В. Цыганова \\ Ульяновский государственный университет, \\ факультет математики, информационных и авиационных технологий, \\ Россия, 432017, Ульяновск, ул. Льва Толстого, 42.
}

\begin{abstract}
Аннотация
Мультисенсорная система фильтрации характеризована математически как результат решения задачи синтеза многомерной дискретной системы фильтрации одного сигнала по данным от множества разнородных датчиков (сенсоров). В стационарной постановке этой задачи приведены три варианта ее решения: Колмогорова-Винера, Калмана в ковариационной форме и Калмана в информационной форме. Осуществлен переход к постановке этих задач в условиях параметрической неопределенности. В целях реализации активного принципа адаптации найден метод формирования инструментального функционала качества для эквивалентной замены недоступного исходного функционала качества среднеквадратической ошибки фильтрации. Показано, что эта замена создает возможность применять для адаптации системы весь аппарат и средства практических методов оптимизации, прежде всего, методов градиентного и ньютоновского типов.

Предложенное теоретическое решение задачи формирования инструментального функционала качества осуществимо при достаточно общих условиях исходной задачи синтеза многомерной дискретной системы фильтрации при бесконечном времени наблюдения.

Выявлено следующее:

- Достаточно сложные операции одношагового предсказания и затем обновления оценок в двухэтапном алгоритме фильтрации целесообразно выполнять в центре принятия решений; здесь же должны выполняться вычислительные операции по минимизации инструментального функционала качества.

- Несложные операции адаптивного масштабирования данных целесообразно оставить в местах нахождения сенсоров.
\end{abstract}

\section{Научная статья}

(2)(7) Контент публикуется на условиях лицензии Creative Commons Attribution 4.0 International (https://creativecommons.org/licenses/by/4.0/deed.ru)

\section{Образец для цитирования}

С ем ушин И. В., Цыг ан о в Ю. В. Активная адаптация распределенной мультисенсорной системы фильтрации // Вестн. Сам. гос. техн. ун-та. Сер. Физ.-мат. науки, 2019. T. 23, № 4. C. 724-743. doi: 10.14498/vsgtu1704.

\section{Сведения об авторах}

Иннокентий Васильевич Семушин (D) https://orcid.org/0000-0002-3687-1110 доктор технических наук, профессор; профессор каф. информационных технологий; e-mail: kentvsem@yandex.ru

Юлия Владимировна Цыганова (1) https://orcid.org/0000-0001-8812-6035

доктор физико-математических наук, доцент; профессор каф. информационных технологий; e-mail: tsyganovajv@gmail.com 
- Алгоритмы адаптации могут быть реализованы для базовых алгоритмов фильтрации, взятых в различных формах: в форме фильтра Колмогорова-Винера, в ковариационной форме фильтра Калмана или в информационной форме фильтра Калмана.

- Вычислительные операции по минимизации инструментального функционала качества целесообразно разрабатывать как варианты реализации современных практических методов оптимизации различного уровня сложности.

Ключевые слова: активная адаптация, мультисенсорная система, распределенная фильтрация Калмана, инструментальный критерий качества оптимизации.

Получение: 18 мая 2019 г. / Исправление: 19 октября 2019 г. / Принятие: 11 ноября 2019 г. / Публикация онлайн: 16 декабря 2019 г.

Введение. Мультисенсорная (многоканальная) задача выделения полезной информации о состоянии сложного объекта не является принципиально новой [1]. Наглядным примером может служить любая комплексная навигационная система. Построение фильтра, выделяющего информацию о движении объекта из данных не одного, а многих разнородных измерителей, позволяет не только обеспечивать критически важное свойство наблюдаемости системы «объект+сенсоры», но - что важнее - получать существенный выигрыш в точности по сравнению с односенсорной системой. В последнее время эта задача получила развитие, связанное с пространственным разнесением сенсоров, - возможностью децентрализации вычислений. Поднимаются новые вопросы: какую часть таких вычислений целесообразно передавать аппаратуре, размещенной в местах нахождения сенсоров (MHC), а какую оставлять в центре принятия решений (ЦПР)? Как согласовывать между собой обработку информации «на местах» и в центре? Как могут отдельные сенсоры взаимодействовать между собой, чтобы улучшать генеральный показатель качества решения задачи оценивания состояний наблюдаемого и/или управляемого объекта?

В математическом смысле синтез стационарного оптимального фильтра в мультисенсорном классе линейных систем является частным случаем общей задачи Колмогорова-Винера при бесконечном времени работы системы. Показателем качества решения служит сумма дисперсий ошибок всех каналов фильтрации $J_{e}$, где $e$ - вектор ошибок (разность желаемого сигнала и выхода фильтра) [1, с. 218]. Классический подход рассматривает $J_{e}$ как исходный функционал качества (ИФК) от передаточных функций $G(z)$ многоканальной системы дискретных фильтров: $J_{e}=J_{e}[G(z)]$. Многомерный фильтр $G_{\star}(z)$ называют оптимальным, если он минимизирует ИФК: $G_{\star}(z) \triangleq \operatorname{argmin}_{G(z)} J_{e}[G(z)]$. Задача отыскания $G_{\star}(z)$ является вариационной задачей, и ее решение хорошо известно [1, с. 219-253].

Теория фильтрации Калмана учитывает эффекты нестационарности и конечности времени работы фильтров. Вывод алгоритма фильтрации Калмана в этой более общей теории базируется на байесовской постановке задачи оценивания, где за показатель качества принимают совместную плотность распределения вероятностей (ПРВ) измерений и оцениваемой величины и макси- 
мизируют эту функцию плотности, что выражает собой «приниип безусловного максимума правдоподобия» [2, с. 51]. Когда условная ПРВ оцениваемой величины при учете полученных измерений является гауссовской плотностью, то оптимальная оценка дается условным математическим ожиданием, поскольку гауссовская ПРВ достигает максимума именно в точке среднего значения [3, с. 217]. Отметим, что байесовская задача в этом случае снова сводится к минимизации среднеквадратического функционала [4, с. 179].

Фильтр Калмана особо важен в стационарном случае: (1º) параметры задачи постоянны, $\left(2^{\circ}\right)$ модель стохастического сигнала (объекта) обладает свойством стабилизируемости, $\left(3^{\circ}\right)$ добавление к ней сенсоров придает этой системе свойство наблюдаемости, $\left(4^{\circ}\right)$ сенсоры обладают ненулевыми погрешностями и $\left(5^{\circ}\right)$ время наблюдения бесконечно. В этом случае решение задачи Калмана совпадает с решением задачи Колмогорова-Винера [4, с. 185].

Наряду с классическим фильтром Калмана в настоящее время разработаны различные методы калмановской фильтрации для распределенных мультисенсорных систем. Наиболее известными являются параллельный информационный фильтр [5], распределенный информационный фильтр [6], распределенный фильтр Калмана с консенсусным фильтром [7], распределенный фильтр Калмана со взвешенным усреднением [8].

Термин «распределенная фильтрация» означает, что средства измерения разнесены в пространстве. В распределенной сети сенсоров может присутствовать ЦПР, в котором вычисляется оптимальная оценка вектора состояния системы. Отдельные сенсоры (узлы сети) могут обмениваться данными как с ЦПР, так и со своими соседями в соответствии с топологией сети, которая может быть задана заранее либо может изменяться во времени.

Задача децентрализованной фильтрации Калмана отличается тем, что в ней отсутствует ЦПР, а каждый сенсор содержит свой локальный процессор для вычисления оптимальной оценки вектора состояния. Эта задача впервые была решена в [5] в 1979 г., а также ее независимое решение представлено в [9]. Подробный обзор современных методов распределенной фильтрации Калмана можно найти в [10]. В настоящее время теория распределенной фильтрации Калмана для мультисенсорных систем продолжает активно развиваться, о чем свидетельствует большое число недавних публикаций, среди которых отметим [11-15].

Однако известные решения указанных задач опираются на полное знание моделей и числовых значений параметров, характеризующих движение объекта и свойства сенсоров. Такое знание возможно лишь теоретически. Его отсутствие на практике вынуждает: либо $(A)$ удовлетворяться субоптимальными решениями, либо $(B)$ прибегать к адаптивным решениям, либо $(C)$ имитировать более сложные (высокоинтеллектуальные) действия человека по извлечению недостающих знаний (или по компенсации их неполноты) в классе самоорганизующихся - самооптимизирующихся - систем.

Предпринятое исследование мотивировано целями $(B)$ и $(C)$. Их может объединить вопрос данной статьи: как применять активный npuнuип aдаnmaции (самооптимизации) к мультисенсорной системе фильтрации? Пассивный принцип адаптации означает опору на предварительное оценивание неизвестных параметров с тем, чтобы затем эти оценки подставлять в классические решения задач фильтрации в пассивном ожидании, что это даст поло- 
жительный эффект.

Активный же принцип адаптации (АПА) означает критериальную самооптимизацию системы [16]. Средняя норма ошибок фильтрации как исходный функционал качества (ИФК) не может служить инструментом самооптимизации. АПА ищет ответ на вопрос: как построить такой вспомогательный функционал качества (ВФК), который эквимодален ИФК, но доступен как инструмент самооптимизации?. При этом эквимодалъность ИФК и ВФК означает: аргументы, доставляющие им минимум, совпадают, и поэтому их минимальные значения достигаются синхронно.

Раздел 1 содержит строгие формулировки решаемой задачи. В разд. 2 приведен компактный вид оптимального фильтра Колмогорова-Винера, чтобы обособить ту часть вычислений, которая одинакова для всех каналов. Раздел 3 дает запись этого решения в ковариационной форме фильтра Калмана и явный вид решения алгебраического уравнения Риккати, что позволяет знать заранее те пределы, к которым должны сходиться соответствующие характеристики адаптивного фильтра. Информационный фильтр Калмана приведен в разд. 4 ввиду перспективы использования в режиме адаптации. Раздел 5 конкретизирует модель адаптивного фильтра и алгоритмы ее оптимизации. В разд. 6 сформирован вспомогательный функционал качества, позволяющий реализовать алгоритмы, приведенные в разд. 5. Численный пример помещен в разд. 7 для экспериментального подтверждения полезности найденного решения. Заключение сообщает, какие выводы дает и какие возможности открывает эта работа.

\section{1. Характеристики задачи: исходные предположения и формаль-} ные обозначения. Мультисенсорность означает, что измеряемые сигналы $\left\{y^{(i)}(t) \mid i=\overline{1, m}\right\}$ от $m>1$ сенсоров находятся в функциональной зависимости от одного полезного сигнала $x(t)$, где $t$-непрерывное время [1, с. 223]. Практическим примером может быть обработка данных множества радиодопплеровких измерителей скорости движения летательного аппарата (ЛА). В этом контексте скорость движения ЛА является тем полезным сигналом $x(t)$, который нужно оценивать по данным измерений $y_{i}(k T)$, доступным от $i=1,2, \ldots, m$ сенсоров в дискретные моменты времени $t_{k} \triangleq k T, k=1,2, \ldots$, где $T$ обозначает заданный темп измерений.

Типична ситуация, когда $x(t)$ моделирует не саму скорость, а отклонение скорости от некоторого известного (программного) режима движения объекта. Эти отклонения вызваны реальными возмущениями со стороны той стохастической среды, в которой происходит физическое движение. Для ЛА это - воздушная среда, для морского объекта - водная среда. Ситуация, когда среда стационарна (нормальный режим функционирования), дает основание принять первое базовое предположение: полезный сигнал $x(t)$ - стационарный случайный процесс с нулевым средним значением и корреляционной функцией $R_{x x}(\tau)$.

Второе базовое предположение задачи фильтрации характеризует сенсоры - источники первичной (измерительной) информации. Исходим из типовых предположений о том, что погрешности $v_{i}(k T)$ присутствуют в измерениях аддитивно:

$$
y_{i}(k T)=x(k T)+v_{i}(k T), \quad i=1,2, \ldots, m,
$$


что они не коррелированы между собой и с полезным сигналом и что сенсоры работают с таким темпом $T$ дискретизации времени, который позволяет применять для их погрешностей гипотезу дискретного белого шума с нулевым средним значением $\mathrm{E}\left\{v_{i}(k T)\right\}=0$ и с ненулевыми дисперсиями $B_{\star, i}^{2}$ :

$$
\mathrm{E}\left\{v_{i}(k T) v_{j}(l T)\right\}=\left\{\begin{aligned}
B_{\star, i}^{2}, & i=j \& k=l, \\
0, & i \neq j \vee k \neq l .
\end{aligned}\right.
$$

Обозначим векторы поступающих данных и ошибок наблюдений в момент $k T$ как $y(k T)$ и $v(k T)$ :

$$
\left.\begin{array}{rl}
y(k T) & \triangleq\left[y_{1}(k T)\left|y_{2}(k T)\right| \cdots \mid y_{m}(k T)\right]^{\top}=H x(k T)+v(k T), \\
H & =\left[\begin{array}{c|c|c|c|}
1 & 1 & \cdots & 1
\end{array}\right]^{\top}, \\
v(k T) & =\left[v_{1}(k T)\left|v_{2}(k T)\right| \cdots \mid v_{m}(k T)\right]^{\top}, \\
R_{\star} & =\mathrm{E}\left\{v(k T) v^{\top}(k T)\right\}=\operatorname{diag}\left[B_{\star, 1}^{2}\left|B_{\star, 2}^{2}\right| \cdots \mid B_{\star, m}^{2}\right] .
\end{array}\right\}
$$

Введем составной вектор, который объединяет всю историю наблюдений, доступных к настоящему моменту времени $k T$, и обозначим его как

$$
Y(k T) \triangleq\left[y(k T)^{\top}\left|y(k T-T)^{\top}\right| \cdots \mid y(k T-j T)^{\top}\right]_{j \rightarrow \infty}^{\top} .
$$

Это случайный вектор растущей со временем размерности $m \times(j+1)$ $j=0,1, \ldots$, чье реализованное значение обозначим соответственно:

$$
Y_{k} \triangleq\left[y_{k}^{\top}\left|y_{k-1}^{\top}\right| \cdots \mid y_{k-j}^{\top}\right]_{j \rightarrow \infty}^{\top}
$$

Предполагаем (для определенности), что для $x(t)$ приемлема $R_{x x}(\tau)$ в простом виде:

$$
R_{x x}(\tau)=A_{\star}^{2} e^{-\alpha_{\star}|\tau|}
$$

чтобы в последующем анализе суть решения оказалась проявлена не в технических деталях, вызванных более сложной моделью, а в принципиальных особенностях мультисенсорной постановки. Этой функции (3) соответствует следующая дискретная спектральная плотность сигнала [1, с. 232]:

$$
\Phi_{x x}(z) \triangleq \mathscr{Z}_{I I}\left\{R_{x x}(\tau)\right\}=\sum_{k=-\infty}^{\infty} z^{-k} R_{x x}(k T)=\frac{A_{\star}^{2}\left(1-d_{\star}^{2}\right)}{\left(1-d_{\star} z\right)\left(1-d_{\star} z^{-1}\right)},
$$

где $d_{\star} \triangleq e^{-\alpha_{\star} T}, z=e^{p T}$ - переменная $z$-преобразования, $p$ - переменная преобразования Лапласа, $\mathscr{Z}_{I I}\{\cdot\}$ обозначает двустороннее $z$-преобразование, применяемое к $\{\cdot\}$, в данном случае к $\left\{R_{x x}(\tau)\right\}$.

Перейдем к бесскобочным обозначениям $x_{k} \triangleq x(k T), y_{k}^{(i)} \triangleq y_{i}(k T), y_{k} \triangleq$ $y(k T)$ и т. п. для функций дискретного времени $k T$ и будем применять соответствующие им односторонние $z$-преобразования

$$
\begin{aligned}
x(z) & \triangleq \mathscr{Z}_{I}\{x(k T)\}=\sum_{j=0}^{\infty} z^{-j} x_{j}, \\
y^{(i)}(z) & \triangleq \mathscr{Z}_{I}\left\{y_{i}(k T)\right\}=\sum_{j=0}^{\infty} z^{-j} y_{j}^{(i)}, \\
y(z) & \triangleq \mathscr{Z}_{I}\{y(k T)\}=\sum_{j=0}^{\infty} z^{-j} y_{j} .
\end{aligned}
$$


Факторизация $\Phi_{x x}(z)=F_{\star}\left(z^{-1}\right) \Phi_{w w}(z) F_{\star}(z)$ спектральной плотности $(4)$ при задании $\Phi_{w w}=1$ означает, что стационарный процесс $x_{k}$ может быть рассмотрен как сформированный устойчивым дискретным фильтром с передаточной функцией

$$
F_{\star}(z)=\frac{A_{\star} \sqrt{1-d_{\star}^{2}}}{1-d_{\star} z^{-1}}
$$

из дискретного белого шума $w_{k}$ с единичной дисперсией [1, p. 209]. Это означает, что

$$
\left.\begin{array}{c}
x_{k}=d_{\star} x_{k-1}+A_{\star} \sqrt{1-d_{\star}^{2}} w_{k}, \\
y_{k}^{(i)}=x_{k}+v_{k}^{(i)}, \quad i=1,2, \ldots, m .
\end{array}\right\}
$$

В гауссовской трактовке имеем ПРВ в явном виде [3, с. 209, 215]:

$$
\left.\begin{array}{rl}
f_{x(k T) \mid Y(k T-T)}\left(\xi \mid Y_{k-1}\right) & =\frac{1}{\sqrt{2 \pi \dot{p}_{k}^{-}}} \exp \left\{-\frac{\left(\xi-\stackrel{\circ}{x}_{k}^{-}\right)^{2}}{2 \dot{p}_{k}^{-}}\right\}, \\
& (\text {первый этап - прогноз на } 1 \text { шаг), } \\
f_{x(k T) \mid Y(k T)}\left(\xi \mid Y_{k}\right) & =\frac{1}{\sqrt{2 \pi \dot{p}_{k}^{+}}} \exp \left\{-\frac{\left(\xi-\stackrel{\circ}{x}_{k}^{+}\right)^{2}}{2 \dot{p}_{k}^{+}}\right\}, \\
\text {(второй этап - обновление), }
\end{array}\right\}
$$

где присутствуют: $\stackrel{x}{x}_{k}^{-}$- оптимальное предсказание значения $x_{k}$, сформированное сразу после учета измерения $y_{k-1}$ вперед $\kappa$ nриходу измерения $y_{k} ; \grave{p}_{k}^{-}-$ дисперсия ошибки оценки $\stackrel{\circ}{x}_{k}^{-} ; \stackrel{\circ}{x}_{k}^{+}$- оптимальная оценка значения $x_{k}$, обновленная в момент прихода и учета $y_{k} ; \stackrel{\circ}{p}_{k}^{+}$- дисперсия ошибки оценки $\stackrel{\circ}{x}_{k}^{+}$. Как отмечено выше, для их вычисления нужно иметь полное знание всех параметров: $\left\{d_{\star}, A_{\star}^{2}, B_{\star, i}^{2} \mid i=1,2, \ldots, m\right\}$. Если их не имеем, то возможны лишь субоптимальные (или адаптивные) оценки, которые будем обозначать, соответственно, так: $\hat{x}_{k}^{-}$вместо $\stackrel{\circ}{x}_{k}^{-} ; \hat{p}_{k}^{-}$вместо $\stackrel{\circ}{p}_{k}^{-} ; \hat{x}_{k}^{+}$вместо $\stackrel{\leftrightarrow}{x}_{k}^{+} ; \hat{p}_{k}^{+}$вместо $\stackrel{\circ}{p}_{k}^{+}$. Они будут возникать из-за того, что вместо точных $\left\{d_{\star}, A_{\star}^{2}, B_{\star, i}^{2} \mid i=1,2, \ldots, m\right\}$ приходится применять неточные значения $\left\{d, A^{2}, B_{i}^{2} \mid i=1,2, \ldots, m\right\}$, чем и вызываются субоптимальные (или адаптивные) значения параметра фильтра, обозначаемые обобщенно как $\theta$ вместо его оптимального значения $\dot{\theta}_{\star}$. Субоптимальные (или адаптивные) оценки ставятся в зависимость от $\theta$ так же, как ИФК, в роли которого равноправно пригоден любой из среднеквадратических критериев:

$$
\begin{aligned}
& J_{e}^{-}(\theta) \triangleq E\left\{\left[\hat{e}_{k}^{-}(\theta)\right]^{2}\right\} \text { или } J_{e}^{+}(\theta) \triangleq E\left\{\left[\hat{e}_{k}^{+}(\theta)\right]^{2}\right\} \text {, } \\
& \hat{e}_{k}^{-}(\theta) \triangleq x_{k}-\hat{x}_{k}^{-}(\theta) \text { или } \hat{e}_{k}^{+}(\theta) \triangleq x_{k}-\hat{x}_{k}^{+}(\theta) \text {. }
\end{aligned}
$$

2. Задача Колмогорова-Винера в мультисенсорной постановке. Минимизация критерия $J_{e}^{+}(\theta)=J_{e}^{+}[G(z)]$, который сейчас определяется как функционал от вектора $G(z)=\left[G^{(1)}(z)\left|G^{(2)}(z)\right| \cdots \mid G^{(m)}(z)\right]^{\top}$ передаточных функций $m$-канальной системы с выходом $\hat{x}^{+}(z) \triangleq G(z)^{\top} y(z)$, дает оптимальное значение $G_{\star}(z)=\left[G_{\star}^{(1)}(z)\left|G_{\star}^{(2)}(z)\right| \cdots \mid G_{\star}^{(m)}(z)\right]^{\top}$ с передаточными 
функциями $G_{\star}^{(i)}(z)=\stackrel{\circ}{a}_{\star}^{(i)} /\left[1-\stackrel{\circ}{\mu}_{\star} z^{-1}\right], i=1,2, \ldots, m$ для формирования оптимальной оценки $\hat{x}^{+}(z)=G_{\star}(z)^{\top} y(z)$, причем коэффициент $\stackrel{\circ}{a_{\star}^{(i)}}$ в числителе $i$-той передаточной функции индивидуален, а коэффициент $\stackrel{\varkappa}{\star}_{\star}$ в знаменателе у всех передаточных функций один и тот же [1, с. 241-245]:

$$
\left.\begin{array}{c}
\stackrel{\circ}{\star}_{\star}^{(i)}=K_{i}^{\star} \stackrel{\circ}{\star}_{\star}, \quad K_{i}^{\star} \triangleq B_{\star}^{2} / B_{\star, i}^{2}, \quad B_{\star}^{2} \triangleq 1 / \sum_{l=1}^{m}\left(1 / B_{\star, l}^{2}\right), \quad \sum_{l=1}^{m} K_{i}^{\star}=1, \\
\stackrel{\circ}{\star}_{\star}=1-\stackrel{\circ}{\mu}_{\star} d_{\star}^{-1}, \quad \stackrel{\circ}{\mu}_{\star} \triangleq e^{-\stackrel{\circ}{\star}_{\star} T}, \quad \operatorname{ch} \stackrel{\circ}{\alpha}_{\star} T=\operatorname{ch} \alpha_{\star} T+\frac{A_{\star}^{2}}{B_{\star}^{2}} \operatorname{sh} \alpha_{\star} T .
\end{array}\right\}
$$

Соответственно, во временно́й области получаем частные оценки $\stackrel{\stackrel{x}{x}}{k}^{+(i)}$ и итоговую оценку $\stackrel{\circ}{x}_{k}^{+}$:

$$
\left.\begin{array}{ll}
(9)^{a} & \stackrel{\circ}{x}_{k}^{+(i)}=\stackrel{\circ}{\mu}_{\star} \stackrel{\circ}{x}_{k-1}^{+(i)}+\stackrel{\circ}{a}_{\star}^{(i)} y_{k}^{(i)}, \quad i=1,2, \ldots, m, \\
(9)^{b} & \stackrel{\circ}{x}_{k}^{+} \triangleq \sum_{i=1}^{m} \stackrel{\circ}{x}_{k}^{+(i)}=\stackrel{\circ}{\mu}_{\star} \stackrel{\circ}{x}_{k-1}^{+}+\stackrel{\circ}{\star}_{\star} \sum_{i=1}^{m} K_{i}^{\star} y_{k}^{(i)} .
\end{array}\right\}
$$

Это решение представим оптимальной моделью $\mathfrak{M}\left(\dot{\theta}_{\star}\right)(10)$ и ее векторпараметр обозначим $\stackrel{\circ}{\star}_{\star},(10)^{g}$ :

$$
\begin{aligned}
& (10)^{a} \quad \stackrel{\circ}{x}_{k}^{+}=\stackrel{\circ}{\mu}_{\star} \stackrel{ }{x}_{k-1}^{+}+\stackrel{\circ}{a}_{\star} y_{k}^{\star}, y_{k}^{\star} \triangleq \sum_{i=1}^{m} y_{k}^{(\star, i)}, \\
& y_{k}^{\star}=x_{k}+v_{k}^{\star}, v_{k}^{\star}=\sum_{i=1}^{m} K_{i}^{\star} v_{k}^{(i)}, \\
& (10)^{b} \quad y_{k}^{(\star, i)} \triangleq K_{i}^{\star} y_{k}^{(i)}, \\
& (10)^{c} \quad \stackrel{\circ}{\mu}_{\star} \triangleq e^{-\stackrel{\circ}{\alpha}_{\star} T}, \quad \operatorname{ch} \stackrel{\circ}{\alpha}_{\star} T=\operatorname{ch} \alpha_{\star} T+\frac{A_{\star}^{2}}{B_{\star}^{2}} \operatorname{sh} \alpha_{\star} T, \\
& (10)^{d} \quad \stackrel{\circ}{a}_{\star}=1-\stackrel{\circ}{\mu}_{\star} d_{\star}^{-1}, \\
& (10)^{e} \quad B_{\star}^{2} \triangleq 1 / \sum_{l=1}^{m}\left(1 / B_{\star, l}^{2}\right), \quad B_{\star}^{2}=\mathrm{E}\left\{\left[v_{k}^{\star}\right]^{2}\right\}, \\
& (10)^{f} \quad K_{i}^{\star} \triangleq B_{\star}^{2} / B_{\star, i}^{2}, \quad \sum_{l=1}^{m} K_{i}^{\star}=1, \\
& (10)^{g} \quad \stackrel{\circ}{\theta}_{\star} \triangleq\left\{\stackrel{\circ}{\mu}_{\star}, \stackrel{\circ}{\star}_{\star}, B_{\star, i}^{2} \mid i=1,2, \ldots, m\right\}=\underset{\hat{x}_{k}^{+}}{\operatorname{argmin}} J_{e}^{+}(\theta), \\
& (10)^{h} \min \left[J_{e}^{+}(\theta)\right]_{\hat{x}_{k}^{+}}=B_{\star}^{2} \stackrel{\circ}{\star}_{\star} \triangleq J_{e}^{+}\left({\left.\stackrel{\circ}{\theta_{\star}}\right)}^{\prime}=\mathrm{E}\left\{\left[\stackrel{\circ}{e}_{k}^{+}\right]^{2}\right\},\right. \\
& \stackrel{\circ}{e}_{k}^{+} \triangleq x_{k}-\stackrel{\circ}{x}_{k}^{+}(\theta) \text {. }
\end{aligned}
$$

ЗАмечАниЕ 1. В теории синтеза стационарных фильтров при бесконечном времени наблюдения (задача Колмогорова-Винера для многомерных 
систем) решение отыскивают в $m$-канальной системе [1, рис. 5.5, с. 223], где каждое $i$-тое измерение $y_{k}^{(i)}$ проходит обработку в $i$-том фильтре непосредственно в МНС $i$-того сенсора для генерирования частной оценки $\stackrel{\circ}{x}_{k}^{+(i)}(9)^{a}$. Затем частные оценки суммируют для получения итоговой оценки $\stackrel{\leftrightarrow}{x}_{k}^{+}(9)^{b}$. В отличие от этого модель $\mathfrak{M}\left(\AA_{\star}\right)(10)$ применяет единственный фильтр $(10)^{a}$ в ЦПР. В МНС $i$-того сенсора остается простая операция масштабирования данных: умножение измеренной величины на весовой коэффициент $K_{i}^{\star}$ по формуле $(10)^{b}$ для передачи в ЦПР. Вычисления в ЦПР сводятся к суммированию переданных данных для образования эквивалентного входа $y_{k}^{\star},(10)^{a}$, и к его обработке в единственном фильтре по первой формуле $(10)^{a}$. Это делает модель $\mathfrak{M}\left(\stackrel{\circ}{\star}_{\star}\right)$ практичной, значительно снижающей общий объем вычислений.

Уравнения $(10)^{a},(10)^{b}$ определяют параметрический вид фильтра Колмогорова-Винера для данных условий. Если $\theta \triangleq\left\{\mu, a, B_{i}^{2} \mid i=1,2, \ldots, m\right\}$, то получим условное обозначение $\mathfrak{M}(\theta)$ этой субоптимальной (или адаптивной) структуры. Если, варьируя этот $\theta$, минимизировать ИФК $J_{e}^{+}(\theta) \triangleq \mathrm{E}\left\{\left[\hat{e}_{k}^{+}(\theta)\right]^{2}\right\}$ (7), то результатом будет параметр $\stackrel{\circ}{\star}_{\star} \triangleq\left\{\stackrel{\circ}{\mu}_{\star}, \stackrel{\circ}{\star}_{\star}, B_{\star, i}^{2} \mid i=1,2, \ldots, m\right\},(10)^{g}$, определяемый через посредство формул $(10)^{c}-(10)^{f}$ и доставляющий этому ИФК минимальное значение $(10)^{h}$.

\section{3. Задача Калмана в мультисенсорной постановке: ковариацион-} ная форма. Согласно «приниипу безусловного максимума правдоподобия» (см. разд. 1), искомые оптимальные оценки определены теоретически как первый и второй моменты соответствующих ПРВ (6):

$$
\begin{array}{ll}
(11)^{a} & \stackrel{\circ}{x}_{k}^{-} \triangleq \mathrm{E}\left\{x(k T) \mid Y(k T-T)=Y_{k-1}\right\}, \\
(11)^{b} & \stackrel{\circ}{p}_{k}^{-} \triangleq \mathrm{E}\left\{\left[x(k T)-\stackrel{\circ}{x}_{k}^{-}\right]^{2} \mid Y(k T-T)=Y_{k-1}\right\}, \\
(11)^{c} & \stackrel{\circ}{x}_{k}^{+} \triangleq \mathrm{E}\left\{x(k T) \mid Y(k T)=Y_{k}\right\}, \\
(11)^{d} & \stackrel{p}{p}_{k}^{+} \triangleq \mathrm{E}\left\{\left[x(k T)-\stackrel{\circ}{x}_{k}^{+}\right]^{2} \mid Y(k T)=Y_{k}\right\} .
\end{array}
$$

Их практическое вычисление составляет алгоритм Калмана [3, с. 217]. Запишем его в ковариационной форме для данной задачи как два чередующихся этапа (12) и (13):

Эman $I-$ прогноз от $(k-1) T$ к моменту $k T, k=1,2, \ldots, ; \stackrel{\circ}{x}_{0}^{+} \triangleq \mathrm{E}\left\{x_{0}\right\}$, $\stackrel{\circ}{p}_{0}^{+} \triangleq \mathrm{E}\left\{\left(x_{0}-\stackrel{\circ}{x}_{0}^{+}\right)^{2}\right\}$ :

$$
\left.\begin{array}{ll}
(12)^{a} & \stackrel{\circ}{x}_{k}^{-}=d_{\star} \stackrel{\circ}{x}_{k-1}^{+}, \\
(12)^{b} & \stackrel{\circ}{p}_{k}^{-}=d_{\star}^{2} \stackrel{\circ}{p}_{k-1}^{+}+A_{\star}^{2}\left(1-d_{\star}^{2}\right) .
\end{array}\right\}
$$

Эman $I I$ - обновление в момент $k T, k=1,2, \ldots, \quad$ благодаря измерению $y_{k}$ :

$$
\left.\begin{array}{ll}
(13)^{a} & \stackrel{\circ}{x}_{k}^{+}=\stackrel{\circ}{x}_{k}^{-}+\stackrel{\circ}{K}_{k}\left(y_{k}-H \stackrel{\circ}{x}_{k}^{-}\right), \\
(13)^{b} & \stackrel{\circ}{K}_{k}=\stackrel{\circ}{p}_{k}^{-} H^{\top}\left(H \stackrel{\circ}{p}_{k}^{-} H^{\top}+R_{\star}\right)^{-1}, \\
(13)^{c} & \stackrel{\circ}{p}_{k}^{+}=\stackrel{\circ}{p}_{k}^{-}-\stackrel{\circ}{K}_{k} H \stackrel{\circ}{p}_{k}^{-} .
\end{array}\right\}
$$


В начале итераций этого алгоритма по $k=1,2, \ldots$ (при малых значениях $k$ ) фильтр Калмана нестационарен. Однако эффекты начальных условий $\stackrel{\circ}{x}_{0}^{+} \triangleq \mathrm{E}\left\{x_{0}\right\}$ и $\stackrel{p}{0}_{0}^{+} \triangleq \mathrm{E}\left\{\left(x_{0}-\stackrel{\circ}{x}_{0}^{+}\right)^{2}\right\}$, входящих в $(12)$ для $k=1$, при возрастании $k \rightarrow \infty$ исчезают, и фильтр стабилизируется (тем быстрее, чем меньше значение $\left|d_{\star}\right|<1$ ). Строго стационарную (предельную) версию ковариационного алгоритма (12), (13) получим в виде:

Эman $I$ - прогноз оценки сигнала на 1 шаг:

$$
\left.\begin{array}{ll}
(14)^{a} & \stackrel{\circ}{x}_{k}^{-}=d_{\star} \stackrel{\circ}{x}_{k-1}^{+}, \\
(14)^{b} & \stackrel{\circ}{p_{\star}^{-}}=d_{\star}^{2} \stackrel{\circ}{\star}_{\star}^{+}+A_{\star}^{2}\left(1-d_{\star}^{2}\right) .
\end{array}\right\}
$$

Эman II - обновление оценки сигнала благодаря измерению $y_{k}$, при этом $\stackrel{\circ}{K}_{\star}=\left[\stackrel{\circ}{\check{K}_{\star, 1}}\left|\stackrel{\circ}{K}_{\star, 2}\right| \cdots \mid \stackrel{\circ}{K}_{\star, m}\right]:$

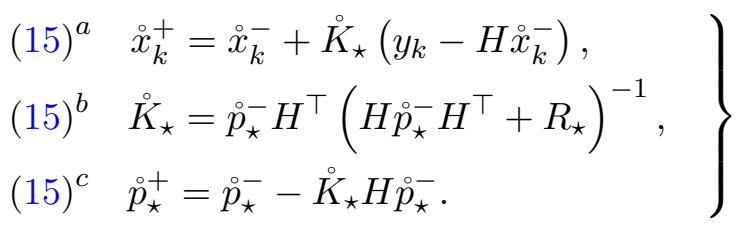

Подстановка выражений $(15)^{b}$ в $(15)^{c}$ и затем в $(14)^{b}$ даст формальное алгебраическое уравнение Риккати (или Лурье, как его называют в [4, с. 185]), но выписывать его нет смысла, поскольку решать его можно лишь многочисленными итерациями по $k$ в алгоритме $(12)^{b},(13)^{b},(13)^{c}$, пока процесс не стабилизируется по значению $p_{k}^{-}$. Обнаружив стабилизацию, считают, что процесс сошелся: $\stackrel{\circ}{p}_{k}^{-} \rightarrow \stackrel{\circ}{p}_{\star}^{-}$и $\stackrel{\circ}{K}_{k} \rightarrow \stackrel{\circ}{K_{\star}}$. При этом выражение $(15)^{c}$ приобретает вид: $\stackrel{\circ}{\star}+_{\star}=\left(1-\sum_{i=1}^{m} \stackrel{\circ}{K}_{\star, i}\right) \stackrel{\circ}{\star}_{\star}^{-}$. Однако явных формул для предельных значений $\stackrel{\circ}{\star}_{\star}^{-}, \stackrel{\circ}{K}_{\star}$ и $\stackrel{\circ}{\star}_{\star}^{+}$таким методом получить невозможно.

Теорема 1. Установивииеся коэфбиииенты Калмана в оптимальном фильтре (14), (15) имеют следующие явные выражения:

$$
\stackrel{\circ}{K}_{\star}=\left[\stackrel{\circ}{K}_{\star, 1}\left|\stackrel{\circ}{K}_{\star, 2}\right| \cdots \mid \stackrel{\circ}{K}_{\star, m}\right]=\stackrel{\circ}{a}_{\star}\left[K_{1}^{\star}\left|K_{2}^{\star}\right| \cdots \mid K_{m}^{\star}\right]
$$

в которых участвуют формулъ: $(10)^{f}$ для $K_{i}^{\star},(10)^{e}$ для $B_{\star}^{2},(10)^{d}$ для $\stackrel{\circ}{\star}_{\star} u$ $(10)^{c} \partial \Omega я \stackrel{\circ}{\mu}_{\star}$.

Доказ ат ельств о. Перепишем $(9)^{a}$ в форме фильтра Калмана. Для этого в $i$-том фильтре Колмогорова-Винера введем обозначение прогнозной

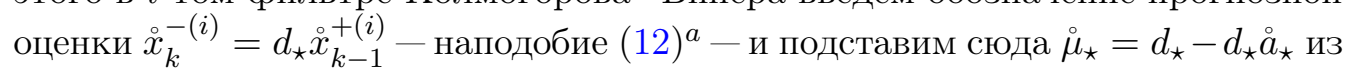
$(10)^{d}$. Учитывая $\stackrel{\circ}{\star}_{\star}^{(i)}=K_{i}^{\star} \stackrel{\circ}{a}_{\star}$ в $(8)$, найдем $\stackrel{\circ}{x}_{k}^{+(i)}=\stackrel{\circ}{x}_{k}^{-(i)}+\stackrel{\circ}{a}_{\star}\left[K_{i}^{\star} y_{k}^{(i)}-\stackrel{\circ}{x}_{k}^{-(i)}\right]$. Суммирование по $i=1,2, \ldots, m$ дает

$$
\stackrel{\circ}{x}_{k}^{+}=\stackrel{\circ}{x}_{k}^{-}+\stackrel{\circ}{a}_{\star}\left[\sum_{i=1}^{m} K_{i}^{\star} y_{k}^{(i)}-\stackrel{\circ}{x}_{k}^{-}\right] .
$$


С первым обозначением в (1) имеем $\sum_{i=1}^{m} K_{i}^{\star} y_{k}^{(i)}=K^{\star} y_{k}$, где

$$
K^{\star} \triangleq\left[K_{1}^{\star}\left|K_{2}^{\star}\right| \cdots \mid K_{m}^{\star}\right] \text {. }
$$

Теперь обратимся к фильтру Калмана, раскрывая скобки в $(15)^{a}$. Зная, что фильтр Колмогорова-Винера является стационарной версией фильтра Калмана, требуем почленного совпадения этих уравнений:

$$
\left.\begin{array}{ll}
(17)^{a} & \stackrel{\circ}{x}_{k}^{+}=\stackrel{\circ}{x}_{k}^{-}+\stackrel{\circ}{a}_{\star} K^{\star} y_{k}-\stackrel{\circ}{a}_{\star} \stackrel{\circ}{x}_{k}^{-}, \\
(17)^{b} & \stackrel{\circ}{x}_{k}^{+}=\stackrel{\circ}{x}_{k}^{-}+\stackrel{\circ}{K}_{\star} y_{k}-\stackrel{\circ}{K}_{\star} H \stackrel{\circ}{x}_{k}^{-} .
\end{array}\right\}
$$

Совпадение $\stackrel{\circ}{a}_{\star} K^{\star}=\stackrel{\circ}{K}_{\star}$ означает $\stackrel{\circ}{\star}_{\star} K_{i}^{\star}=\stackrel{\circ}{K}_{\star, i}$. Это совпадение и свойство суммы $(10)^{f}$ приводят к результату: $\stackrel{\circ}{K}_{\star} H=\stackrel{\circ}{a}_{\star} K^{\star} H=\stackrel{\circ}{a}_{\star} \sum_{i=1}^{m} K_{i}^{\star}=\stackrel{\circ}{a_{\star}}$.

4. Задача Калмана в мультисенсорной постановке: информационная форма. Если строить адаптивный фильтр Калмана на основе ковариационной формы (разд. 3), то вычисления могут оказаться излишне сложными. Приведем теоретически эквивалентное решение в информационной (инверсной относительно предыдущего варианта) форме оптимального фильтра Калмана.

Вводим обозначения инверсных величин:

$$
\grave{\lambda}_{k}^{-} \triangleq 1 / \stackrel{\circ}{p}_{k}^{-}, \quad \grave{\lambda}_{k}^{+} \triangleq 1 / \stackrel{\circ}{p}_{k}^{+}, \quad \stackrel{\circ}{s}_{k}^{-} \triangleq \grave{\lambda}_{k}^{-} \stackrel{\circ}{x}_{k}^{-}, \quad \stackrel{\circ}{s}_{k}^{+} \triangleq \stackrel{\circ}{\lambda}_{k}^{+} \stackrel{\circ}{x}_{k}^{+} .
$$

Тогда из (12) и (13) получаем следующее.

Эman $I$ - прогноз от $(k-1) T$ к моменту $k T, k=1,2, \ldots, ; \stackrel{\circ}{s}_{0}^{+} \triangleq \stackrel{\circ}{\lambda}_{0}^{+} \stackrel{\circ}{x}_{0}^{+}, \grave{\lambda}_{0}^{+} \triangleq$ $1 / \stackrel{\circ}{0}_{0}^{+}$:

$$
\left.\begin{array}{ll}
(18)^{a} \quad \stackrel{\circ}{s}_{k}^{-}=\frac{\stackrel{\circ}{k}_{k}^{-}}{\dot{\lambda}_{k-1}^{+}} d_{\star} \stackrel{\circ}{k-1}_{k-1}^{+}, \\
(18)^{b} \quad \grave{\lambda}_{k}^{-}=\frac{\grave{\lambda}_{k-1}^{+}}{d_{\star}^{2}+\grave{\lambda}_{k-1}^{+} A_{\star}^{2}\left(1-d_{\star}^{2}\right)} .
\end{array}\right\}
$$

Эman II - обновление в момент $k T, k=1,2, \ldots$, благодаря измерению $y_{k}$ :

$$
\left.\begin{array}{ll}
(19)^{a} & \stackrel{\circ}{s_{k}^{+}}=\stackrel{\circ}{s}_{k}^{-}+H^{\top} R_{\star}^{-1} y_{k}=\stackrel{\circ}{s}_{k}^{-}+\Delta \stackrel{\circ}{s}_{k}^{+}, \\
(19)^{b} & \Delta \stackrel{\circ}{s}_{k}^{+} \triangleq \sum_{i=1}^{m} \frac{y_{k}^{(i)}}{B_{\star, i}^{2}}, \quad \Delta \grave{\lambda}_{k}^{+} \triangleq\left(B_{\star}^{2}\right)^{-1}, \\
(19)^{c} & \stackrel{\circ}{\lambda}_{k}^{+}=\stackrel{\circ}{\lambda}_{k}^{-}+\Delta \grave{\lambda}_{k}^{+} .
\end{array}\right\}
$$

Запись стационарной версии информационной формы (18), (19) опускаем как очевидную. Вычислять слагаемые для суммы в $(19)^{b}$ можно в МНС, остальные действия - в ЦПР.

5. Параметрическая неопределенность усложняет задачу. В общем случае априорной неопределенности всех параметров $\left\{d_{\star}, A_{\star}^{2}, B_{\star, i}^{2} \mid i=\right.$ $1,2, \ldots, m\}$ задач Колмогорова-Винера или Калмана их решения (10), или 
(14), (15), или (18), (19) не могут быть реализованы. Вместо каждого из этих оптимальных решений возможно иметь лишь множество аналогов: либо субоптимальных фильтров, либо адаптивных фильтров.

Если любое из оптимальных решений обозначать $\mathfrak{M}\left(\AA_{\star}\right)$, то соответствующее множество субоптимальных (адаптивных) решений следует обозначать как $\mathfrak{M}(\theta)$, где $\theta$ - субоптимальное либо настраиваемое значение параметра. Например, в качестве адаптивной версии фильтра Колмогорова-Винера или, что равнозначно, стационарного фильтра Калмана (в ковариационной форме) следует брать $\mathfrak{M}(\theta)$ в следующем виде:

$$
\left.\begin{array}{ll}
(20)^{a} & \hat{x}_{k}^{-}=d \hat{x}_{k-1}^{+}, \\
(20)^{b} & \hat{x}_{k}^{+}=\hat{x}_{k}^{-}+a\left(K y_{k}-\hat{x}_{k}^{-}\right)
\end{array}\right\} \begin{gathered}
\mathfrak{M}(\theta)-\text { адаптивная модель: } \\
\theta=\left\{a, d, K_{i} \mid i=1,2, \ldots, m\right\} \\
K=\left[K_{1}\left|K_{2}\right| \cdots \mid K_{m}\right] .
\end{gathered}
$$

Каждое пробное значение $\theta:=\theta[n] \in \Theta \subset \mathbb{R}^{m+2}$, где $n=1,2, \ldots$ - номер пробного значения, создает $n$-ную параметрическую версию $\mathfrak{M}(\theta[n])$ субоптимального фильтра $\mathfrak{M}(\theta)(20)$ в некотором допустимом множестве $\Theta$. Каждая $n$-ная версия $\mathfrak{M}(\theta[n])$ формирует свою пару оценок: экстраполяционную $\hat{x}_{k}^{-} \triangleq \hat{x}_{k}^{-}(\theta[n])$ и отфильтрованную $\hat{x}_{k}^{+} \triangleq \hat{x}_{k}^{+}(\theta[n])$, обе страдающие погрешностями: $\hat{e}_{k}^{-}(\theta[n]) \triangleq x_{k}-\hat{x}_{k}^{-}(\theta[n])$ и $\hat{e}_{k}^{+}(\theta[n]) \triangleq x_{k}-\hat{x}_{k}^{+}(\theta[n])$, с соответствующими значениями ИФК: $J_{e}^{-}(\theta[n]) \triangleq \mathrm{E}\left\{\left[\hat{e}_{k}^{-}(\theta[n])\right]^{2}\right\}$ и $J_{e}^{+}(\theta[n]) \triangleq \mathrm{E}\left\{\left[\hat{e}_{k}^{+}(\theta[n])\right]^{2}\right\}$, т. е. значениями дисперсий ошибок (7) в параметрическом пространстве $\Theta$ при работе этой $n$-ной версии на всех наличных данных $y_{j}^{(i)} \triangleq y_{i}(j T), i=$ $1,2, \ldots, m$, где $j=k, k-1, \ldots, l(l \rightarrow-\infty$ теоретически).

Минимизация любого критерия (7) влечет достижение минимума другим критерием из этой пары, поэтому достаточно заниматься одним из них. Однако любой ИФК (7) может быть минимизирован лишь теоретически, так как ошибки оценивания $\hat{e}_{k}^{-}(\theta[n]), \hat{e}_{k}^{+}(\theta[n])$ не являются доступными величинами. Если бы любой критерий $J_{e}^{ \pm}(\theta[n]) \triangleq \mathrm{E}\left\{\left[\hat{e}_{k}^{ \pm}(\theta[n])\right]^{2}\right\}$ из (7) был доступен, то для его численной минимизации в пространстве параметров фильтра (20) можно было бы пытаться применять стандартные методы, например, метод скорейшего спуска [17, p. 22]:

$$
\left.\begin{array}{ll}
(21)^{a} & g(\theta[n])=\nabla_{\theta} J_{e}^{ \pm}(\theta[n]), \\
(21)^{b} & \theta[n+1]=\theta[n]-\gamma[n] g(\theta[n])
\end{array}\right\}
$$

с величиной $n$-ного шага $\gamma[n]$ (хотя это не лучший выбор), или более надежный метод Ньютона [17, p. 44]:

$$
\begin{array}{ll}
(22)^{a} & g(\theta[n])=\nabla_{\theta} J_{e}^{ \pm}(\theta[n]), \\
(22)^{b} & G(\theta[n])=\nabla_{\theta}^{2} J_{e}^{ \pm}(\theta[n]), \\
(22)^{c} & \text { solve } G(\theta[n]) \delta=-g(\theta[n]) \text { for } \delta \triangleq \delta[n], \\
(22)^{d} & \theta[n+1]=\theta[n]+\delta[n],
\end{array}
$$


или другие методы этого типа [17, р. 49-57]. При их сходимости к точке минимума ИФК можно было бы рассчитывать, что $\theta[n] \rightarrow \stackrel{\circ}{\star}_{\star}=\left\{\stackrel{\circ}{a}_{\star}, d_{\star}, K_{i}^{\star} \mid\right.$ $i=1,2, \ldots, m\}$.

ЗАмечАниЕ 2. Существенно, что итерации по номерам версий субоптимального фильтра (при $n=1,2, \ldots$ ) можно выполнять не в реальном, а в ускоренном (компьютерном) темпе времени на одном и том же множестве $y_{j}^{(i)} \triangleq y_{i}(j T), i=1,2, \ldots, m$, где $j=k, k-1, \ldots, l$, экспериментальных данных, если они сохранены для этого: менъшее компъютерное время поиска лучшей версии в обмен на бо́льшие затраты компьютерной памяти.

6. Построение вспомогательного (инструментального) функционала качества. Однако реализация таких идей невозможна. Поскольку в задаче фильтрации ошибки $\hat{e}_{k}^{ \pm}(\theta)$ (вторая строка в $\left.(7)\right)$ не могут быть известны, любой исходный функционал ошибки (первая строка в (7)) не может быть практическим инструментом оптимизации фильтра.

Задача формирования ВФК, эквимодального исходному функционалу качества, поставлена [18] как задача Активного Приниипа Адаптации (АПА).

ОПРеДЕлЕние 1. Два функционала эквимодалънъ друг другу, если совпадают аргументы, доставляющие им минимум, а именно: минимум одного функционала влечет минимум другого функционала; в вычислительном процессе минимизации одного из них, доступного для реализации, минимум другого, не доступного для реализации, достигается автоматически.

Обозначим доступный для реализации ВФК обобщенно как $J_{a}(\theta)$, следуя термину Auxiliary Performance Index. Не доступный для реализации ИФК обозначим $J_{o}(\theta)$ - Original Performance Index. Потребуем свойство:

$$
J_{a}(\theta)=J_{o}(\theta)+\text { const }_{\theta} .
$$

Теорема 2. Пусть за $J_{о}(\theta)$ принят первый из критериев (7) и доступный для реализащии прощесс $\varepsilon_{k}^{-}(\theta)$ определен выражением

$$
\varepsilon_{k}^{-}(\theta) \triangleq \frac{1}{m} \sum_{i=1}^{m} y_{k}^{(i)}-\hat{x}_{k}^{-}(\theta) .
$$

Тогда (23) выполнено для $J_{a}(\theta) \triangleq \mathrm{E}\left\{\left[\varepsilon_{k}^{-}(\theta)\right]^{2}\right\}$ nри $\operatorname{const}_{\theta}=\frac{1}{m^{2}} \sum_{i=1}^{m} B_{\star, i}^{2}$.

Доказательств о. Домножим первое выражение в (1) слева на $H^{\top}$, поделим на $m$ и затем вычтем $\hat{x}_{k}^{-}(\theta)$. Получаем результат:

$$
\varepsilon_{k}^{-}(\theta)=\left[x_{k}-\hat{x}_{k}^{-}(\theta)\right]+\frac{1}{m} \sum_{i=1}^{m} v_{k}^{(i)}=e_{k}^{-}(\theta)+\bar{v}_{k}, \quad \bar{v}_{k} \triangleq \frac{1}{m} \sum_{i=1}^{m} v_{k}^{(i)} .
$$

Возводя (24) в квадрат, осредняя и учитывая $\mathrm{E}\left\{\left[e_{k}^{-}(\theta)\right]\left[\bar{v}_{k}\right]\right\}=0$, находим

$$
\mathrm{E}\left\{\left[\varepsilon_{k}^{-}(\theta)\right]^{2}\right\}=\mathrm{E}\left\{\left[e_{k}^{-}(\theta)\right]^{2}\right\}+\mathrm{E}\left\{\left[\bar{v}_{k}\right]^{2}\right\}, \quad \mathrm{E}\left\{\left[\bar{v}_{k}\right]^{2}\right\}=\frac{1}{m^{2}} \sum_{i=1}^{m} B_{\star, i}^{2} .
$$


Сопоставляя (25) с (23), убеждаемся в справедливости утверждения.

Благодаря этому результату задача активной адаптации применительно к мультисенсорной фильтрации получает инструментальное решение (рис. 1). Это означает, что в практических методах (21) или (22) в качестве рабочего критерия самооптимизации может быть взят $\min _{\theta} \frac{1}{N} \sum_{i=1}^{N}\left[\varepsilon_{k}^{-}(\theta)\right]^{2}$, где $N$ интервал осреднения для приближенного оценивания математического ожидания $J_{a}(\theta) \triangleq \mathrm{E}\left\{\left[\varepsilon_{k}^{-}(\theta)\right]^{2}\right\}$.

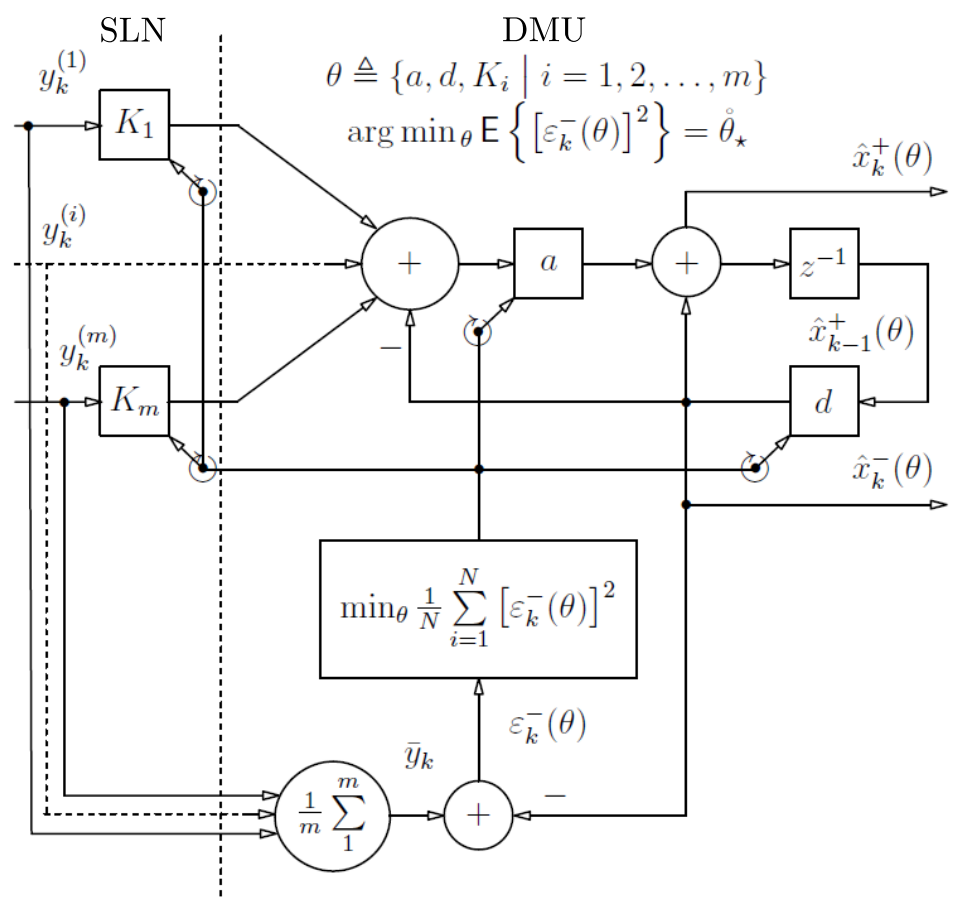

Рис. 1. Активный принцип адаптации фильтра в мультисенсорной постановке: $z^{-1}$ - блок задержки на время такта $T$; SLN - место нахождения сенсоров; DMU - центр принятия решений. Теоретическая сходимость при $n \rightarrow \infty: \theta[n] \rightarrow \stackrel{\circ}{\star}_{\star}=\left\{\stackrel{\circ}{\star}_{\star}, d_{\star}, K_{i}^{\star} \mid i=1,2, \ldots, m\right\}$

[Figure 1. Active principle of adaptation in the multi-sensor problem statement: $z^{-1}-$ a one-step delay unit; SLN - sensors' location in the network; DMU - Decision Making Unit. Theoretical convergence at $\left.n \rightarrow \infty: \theta[n] \rightarrow \stackrel{\circ}{\star}_{\star}=\left\{\stackrel{\circ}{a}_{\star}, d_{\star}, K_{i}^{\star} \mid i=1,2, \ldots, m\right\}\right]$

7. Численный пример. Численные эксперименты проведем в системе MATLAB в режиме двумерной настройки. Для этого считаем, что входные данные содержат сигнал $x(t)$ с двумя неизвестными параметрами $A_{\star}^{2}$ и $\alpha$ корреляционной функции (3). Будем генерировать отсчеты $x_{k}$ сигнала по алгоритму (5), в котором зададим точные значения двух параметров модели: $d_{\star} \triangleq e^{-\alpha_{\star} T}=3 / 5$ и $A_{\star}^{2}=\rho_{\star}^{2} B_{\star}^{2}$. Здесь используем обозначение $\rho_{\star}^{2} \triangleq A_{\star}^{2} / B_{\star}^{2}$ для отношения мощностей "сигнал/шум", где $B_{\star}^{2}$ взято из (8). Для модельного случая четырех сенсоров $(m=4)$ с известными мощностями шумов измерения $B_{\star, i}^{2}=1(i=\overline{1,4})$ имеем $B_{\star}^{2}=1 / 4$, при этом формула (16) Теоремы 1 дает установившиеся значения коэффициентов фильтра Калмана: 
$\stackrel{\circ}{K}_{\star}=\stackrel{\circ}{a}_{\star}[0.25|0.25| 0.25 \mid 0.25]$. Его параметр $\stackrel{\circ}{\star}_{\star}$ удовлетворяет выражению $(10)^{d}$, а $\stackrel{\circ}{\star}_{\star}$ - выражению $(10)^{c}$, которому придадим удобный для вычислений вид:

$$
\begin{aligned}
& \stackrel{\circ}{\mu}_{\star}=\beta_{\star}-\sqrt{\beta_{\star}^{2}-1}, \\
& \beta_{\star} \triangleq(1 / 2)\left[\left(d_{\star}^{-1}+d_{\star}\right)+\rho_{\star}^{2}\left(d_{\star}^{-1}-d_{\star}\right)\right] .
\end{aligned}
$$

Таким образом, адаптивному фильтру (см. рис. 1) неизвестен двумерный $\stackrel{\circ}{\star}_{\star}=\left\{\stackrel{\circ}{a}_{\star}, d_{\star}\right\}$. В адаптивном фильтре его должен заменять настраиваемый параметр $\theta \triangleq\{a, d\}$. Параметры $\left\{K_{i} \mid i=1,2,3,4\right\}$ настраивать не нужно, поскольку их точные значения $\left\{K_{i}^{\star}=0.25 \mid i=1,2,3,4\right\}$ известны.

План вычислительных экспериментов следующий. Будем сохранять $N$ последних значений из серии измерений (2) при значении $\stackrel{\circ}{\star}_{\star}=\left\{\stackrel{\circ}{a}_{\star} ; d_{\star}\right\}$. Зададим интервал измерений $N=500$. Накопленные измерения используем для вычисления значений исходного и вспомогательного функционалов качества $J_{o}(\theta)$ и $J_{a}(\theta)$, заменяя оператор математического ожидания $\mathrm{E}\{\cdot\}$, предполагаемый в ключевом соотношении (23), оператором текущего среднего. Делая это при изменяющихся значениях параметров $a$ и $d$ фильтра для разных значений $\rho_{\star}^{2}$, построим графики.

Результаты, полученные при этих условиях эксперимента, показывают процесс оптимальной фильтрации сигнала на рис. 2 для соотношений сигнал/шум $\rho_{\star}^{2}=1$ (графики слева) и $\rho_{\star}^{2}=100$ (графики справа).

Возможность сопоставить значения ИФК и ВФК при тех же условиях эксперимента дает следующие результаты (рис. 3 и 4). Из рис. 3 видно, что точки минимума $J_{o}(\theta)$ и $J_{a}(\theta)$ по $a$ и $d$ совпадают и соответствуют оптимальным значениям $\stackrel{\circ}{\stackrel{\circ}{\star}_{\star}}=4 / 9 \approx 0.44$ и $d_{\star}=3 / 5=0.6$. Рис. 4 также показывает, что минимумы критериев $J_{o}(\theta)$ и $J_{a}(\theta)$ достигаются в точке оптимальных значений параметров для данного $\rho_{\star}^{2}=100:$ при $\stackrel{\circ}{\star}_{\star}=-808 / 9+(8 / 9) \sqrt{10426} \approx 0.98$ и $d_{\star}=3 / 5=0.6$.

Таким образом, проведенные вычислительные эксперименты подтверждают полученные в работе теоретические результаты.
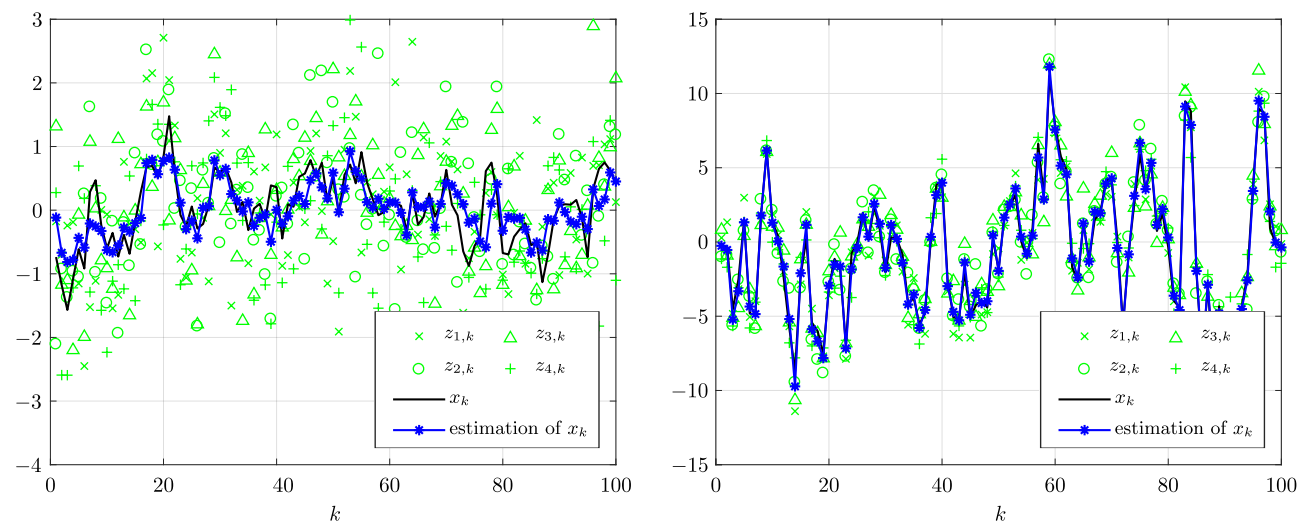

Рис. 2. Оптимальная фильтрация сигнала $x_{k}(k=1, \ldots, 100)$ по данным четырех сенсоров при различных отношениях сигнал/шум: $\rho_{\star}^{2}=1$ (слева) и $\rho_{\star}^{2}=100$ (справа)

[Figure 2. Optimal filtering process of signal $x_{k}(k=1, \ldots, 100)$ given data from the four sensors at different signal-to-noise ratios: $\rho_{\star}^{2}=1$ (left) and $\rho_{\star}^{2}=100$ (right)] 

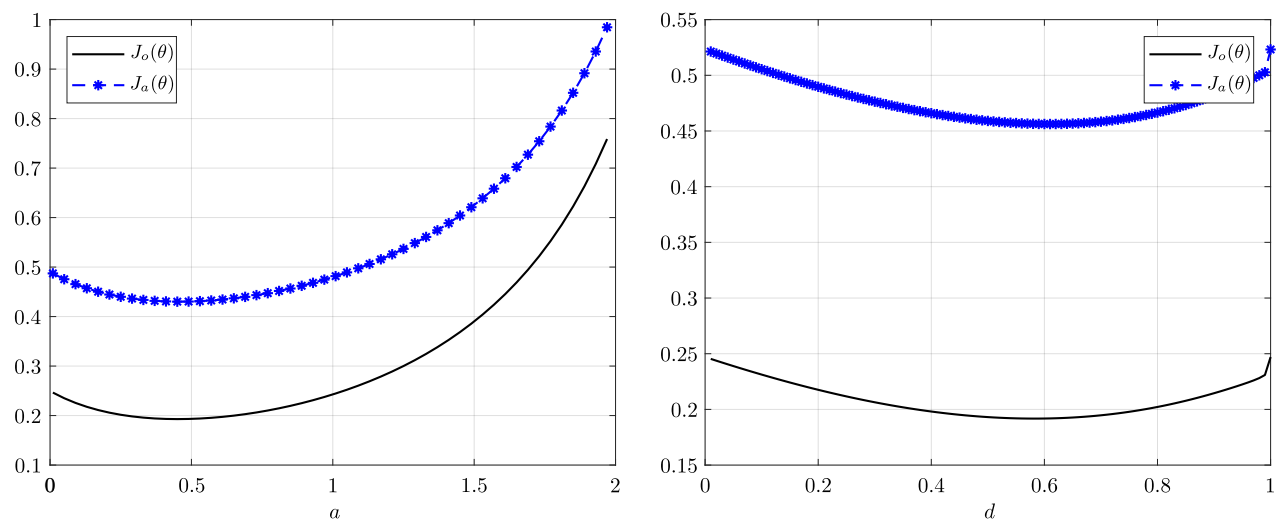

Рис. 3. Значения исходного функционала качества $J_{o}(\theta)$ и вспомогательного функционала качества $J_{a}(\theta)$, вычисленные по $N=500$ данным измерений от четырех сенсоров для отношения сигнал/шум $\rho_{\star}^{2}=1$ при изменении параметров адаптивного фильтра $\theta=\{a, d\}$

[Figure 3. Values of $J_{o}(\theta)$ (OPI - Original Performance Index) and $J_{a}(\theta)$ (API - Auxiliary Performance Index) calculated based on $N=500$ measurement data from four sensors at signalto-noise ratio $\rho_{\star}^{2}=1$ vs the adaptive filter parameter $\left.\theta=\{a, d\}\right]$
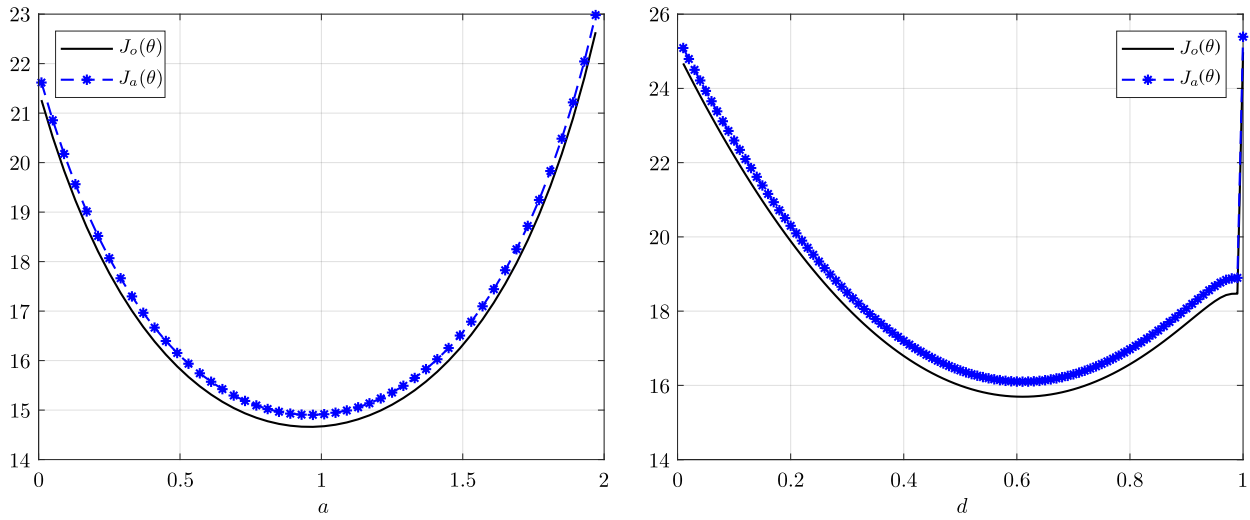

Рис. 4. Значения исходного функционала качества $J_{o}(\theta)$ и вспомогательного функционала качества $J_{a}(\theta)$, вычисленные по $N=500$ данным измерений от четырех сенсоров для отношения сигнал/шум $\rho_{\star}^{2}=100$ при изменении параметров адаптивного фильтра $\theta=\{a, d\}$

[Figure 4. Values of $J_{o}(\theta)$ (OPI - Original Performance Index) and $J_{a}(\theta)$ (API - Auxiliary Performance Index) calculated based on $N=500$ measurement data from four sensors at signalto-noise ratio $\rho_{\star}^{2}=100$ vs the adaptive filter parameter $\left.\theta=\{a, d\}\right]$

Заключение. Установлена возможность формирования и использования инструментального функционала качества для задачи самооптимизации систем мультисенсорного выделения одного полезного сигнала из зашумленных показаний множества датчиков, отличительной особенностью которой является невозможность использования исходного функционала ошибки фильтрации для решения этой задачи.

Данный инструментальный функционал отличается от исходного функционала ошибки фильтрации на постоянную величину, не зависящую от параметров системы обработки поступающей от датчиков измерительной инфор- 
мации. В силу этой связи минимум инструментального функционала влечет минимум исходного функционала, но, в отличие от последнего, может быть найден применением к нему всего аппарата и средств оптимизации.

Предложенное решение осуществимо при достаточно общих условиях:

- модель сигнала ( $\left.\mathfrak{M}_{\text {signal }}\right)$ есть обоснованное представление случайного полезного сигнала в виде стационарного марковского процесса $p$-го порядка как решения линейного устойчивого стохастического дифференциального уравнения $p$-го порядка с неизвестными параметрами уравнения и ковариаций порождающих шумов;

- модель сенсоров ( $\left.\mathfrak{M}_{\text {sensors }}\right)$ есть представление измерительных данных как поступающих дискретно во времени линейных комбинаций сигнала и дискретных белых шумов с неизвестными ковариациями.

В результате детального анализа этого решения на частном примере адаптивного оценивания марковского процесса 1-го порядка выявлено следующее:

- Модель сигнала $\left(\mathfrak{M}_{\text {signal }}\right)$ диктует достаточно сложные операции одношагового предсказания и затем обновления оценок в двухэтапном алгоритме фильтрации; их целесообразно выполнять в одном месте - в центре принятия решений; здесь же должны выполняться вычислительные операции по минимизации инструментального функционала качества.

- Модель сенсоров $\left(\mathfrak{M}_{\text {sensors }}\right)$ диктует несложные операции адаптивного масштабирования данных; их целесообразно оставлять в местах нахождения сенсоров (датчиков первичной измерительной информации).

- Базовые алгоритмы фильтрации нуждаются в придании им алгоритмов адаптации параметров; они могут быть взяты в различных формах: (a) в форме фильтра Колмогорова-Винера, (б) в ковариационной форме фильтра Калмана или (в) в информационной форме фильтра Калмана.

- Вычислительные операции по минимизации инструментального функционала качества должны быть найдены как варианты эффективных алгоритмов различного уровня сложности, изученные, например, в [19].

Теоретическая разработка, предпринятая в данной работе, открывает возможности развития этой темы в следующих направлениях:

1) Применение модели сигнала $\left(\mathfrak{M}_{\text {signal }}\right)$ как процесса порядка $p>1$.

2) Сравнение методов минимизации инструментального функционала качества по вычислительным затратам, скорости сходимости и точности.

3) Анализ эффекта модельных неточностей в системе мультисенсорного выделения сигнала, действующей по активному принципу адаптации.

Конкурирующие интересы. Конкурирующих интересов не имеем.

Авторский вклад и ответственность. Все авторы принимали участие в разработке концепции статьи и в написании рукописи. Авторы несут полную ответственность за предоставление окончательной рукописи в печать. Окончательная версия рукописи одобрена всеми авторами.

Финансирование. Исследование выполнено при финансовой поддержке Российского Фонда Фундаментальных Исследований и Правительства Ульяновской области в рамках научных проектов № 18-41-732002 и № 18-47-730001. 


\section{Библиографический список}

1. Катковник В. Я., Полуэктов Р. А. Многомерные дискретные системы управления. М.: Наука, 1966. 416 с.

2. Балакришнан А. Теория фильтрации Калмана. М.: Мир, 1988. 168 с.

3. Maybeck P. S. Stochastic Models, Estimation, and Control. vol.1/ Mathematics in Science and Engineering. vol. 141. New York: Academic Press, Inc, 1979. xix +423 pp.

4. Фомин В. Н. Рекуррентное оценивание и адаптивная фильтрация. М.: Наука, 1984. 288 c.

5. Speyer J. Computation and transmission requirements for a decentralized linear-quadraticGaussian control problem// IEEE Trans. Automatic Control, 1979. vol. 24, no. 2. pp. 266269. doi: 10.1109/TAC.1979.1101973.

6. Rao B. S., Durrant-Whyte H. F. Fully decentralised algorithm for multisensor Kalman filtering// IEE Proc.-Control Theory Appl., 1991. vol.138, no. 5. pp. 413-420. doi: 10. 1049/ip-d.1991.0057.

7. Olfati-Saber R. Distributed Kalman filtering and sensor fusion in sensor networks / Networked Embedded Sensing and Control/ Lecture Notes in Control and Information Science, 331; eds. P. J. Antsaklis, P. Tabuada. Springer, Berlin, Heidelberg, 2006. pp. 157-167. doi: 10.1007/11533382_10.

8. Alriksson P., Rantzer A. Model based information fusion in sensor networks // IFAC Proceedings Volumes, 2008. vol.41, no.2. pp. 4150-4155. doi: 10.3182/20080706-5-KR-1001. 00698.

9. Rao B. S. Y., Durrant-Whyte H. F., Sheen J. A. A fully decentralized multi-sensor system for tracking and surveillance// Int. J. Robot. Res., 1993. vol. 12, no. 1. pp. 20-44. doi: 10. $1177 / 027836499301200102$.

10. Mahmoud M. S., Khalid H. M. Distributed Kalman filtering: a bibliographic review // IET Control Theory and Applications, 2013. vol.7, no. 4. pp. 483-501. doi: 10.1049/iet-cta. 2012.0732.

11. Marelli D., Zamani M., Fu M., Ninness B. Distributed Kalman filter in a network of linear systems // Systems Control Letters, 2018. vol.116, no.6. pp. 71-77. doi:10.1016/j. sysconle.2018.04.005.

12. Wu Z., Fu M., Xu Yo., Lu R. A distributed Kalman filtering algorithm with fast finite-time convergence for sensor networks // Automatica, 2018. vol. 95, no. 9. pp. 63-72. doi: 10.1016/ j. automatica.2018.05.012.

13. Dormann K., Noack B., Hanebeck U. D. Optimally distributed Kalman filtering with datadriven communication // Sensors, 2018. vol. 18, no. 4. pp. 1034. doi: 10.3390/s18041034.

14. Badyn M. H., Mesbahi M. Large-scale distributed Kalman filtering via an optimization approach // IFAC PapersOnLine, 2017. vol.50, no.1. pp. 10742-10747. doi: 10.1016/j . ifacol.2017.08.2268.

15. Govaers F. Distributed Kalman filter (Chapter 13) / Kalman Filters - Theory for Advanced Applications; eds. Ginalber Luiz de Oliveira Serra. London: IntechOpen, 2018. pp. 253-272. doi: 10.5772/intechopen.71941.

16. Semushin I. V. The APA based time-variant system identification / 53rd IEEE Conference on Decision and Control (15-17 December 2014, Los Angeles, CA, USA), 2014. pp. 41374141. doi : 10.1109/CDC.2014.7040033.

17. Fletcher R. Practical Methods of Optimization. Chichester, Great Britain: John Wiley \& Sons Ltd. xiv+436 pp. doi : 10.1002/9781118723203.

18. Semushin I. V. Adaptation in stochastic dynamic systems - Survey and new results II // Int. J. Communications, Network, and System Sciences, 2011. vol.4, no.4. pp. 266-285. doi: $10.4236 /$ ijens.2011.44032.

19. Цыганова Ю. В. Ортогонализованные блочные методы для параметрической идентификации дискретных линейных стохастических систем: Дис. ... Д-ра физ.-мат. наук: 05.13.18. Ульяновск: Ульяновский государственный университет, 2017. 400 с. 


\title{
MSC: 93E35, 93A30, 65C20
}

\section{Active adaptation of a distributed multi-sensor filtering system}

\author{
I. V. Semushin, Yu. V. Tsyganova \\ Ulyanovsk State University, \\ Faculty of Mathematics, Information and Aviation Technologies, \\ 42, L. Tolstoy st., Ulyanovsk, 432017, Russian Federation.
}

\begin{abstract}
A multi-sensor filtering system is characterized mathematically as a result of the solution to the problem of synthesizing the multi-dimensional discrete system of filtering a single signal from heterogeneous data sources. The stationary problem statement has three variants of its solution: by Kolmogorov-Wiener, Kalman covariance, and Kalman information forms. In the body of the paper, we actualize a problem of these solutions under uncertainty conditions. Aimed at the Active Principle of Adaptation, we have found a method to form an instrumental performance index to substitute the inaccessible original performance index (filtering error mean square) by that criterion functional we created. This substitution makes it possible to apply for system adaptation all apparatus and tools of practical optimization methods, first of all, the gradient and Newton-like methods.

Our findings follow:

- Stretching one-step prediction and measurement update operations are wise to perform at the Decision Making Center; computation operations aimed to minimize the instrumental performance index are to be done in this place, too.

- Uncompounded procedures of adaptive data scaling are advisable to complete at the sensors' location in the network.

- Adaptation algorithms may be implemented based for filter structures taken in different forms: Kolmogorov-Wiener, Kalman covariance, or Kalman information forms.

- Computational operations for minimizing the instrumental performance index would be beneficial to develop as versions to implement the modern practical optimization methods of different levels of complexity.
\end{abstract}

\section{Research Article}

๖ ()(i) The content is published under the terms of the Creative Commons Attribution 4.0 International License (http://creativecommons.org/licenses/by/4.0/)

Please cite this article in press as:

Semushin I. V., Tsyganova Yu. V. Active adaptation of a distributed multi-sensor filtering system, Vestn. Samar. Gos. Tekhn. Univ., Ser. Fiz.-Mat. Nauki [J. Samara State Tech. Univ., Ser. Phys. Math. Sci.], 2019, vol. 23, no. 4, pp. 724-743. doi: 10.14498/vsgtu1704 (In Russian).

\section{Authors' Details:}

Innokentiy V. Semushin (1D https://orcid.org/0000-0002-3687-1110

Dr. Techn. Sci.; Professor; Dept. of Information Technology; e-mail: kentvsem@yandex.ru

Yulia V. Tsyganova (1) https://orcid.org/0000-0001-8812-6035

Dr. Phys. \& Math. Sci.; Professor; Dept. of Information Technology;

e-mail: tsyganovajv@gmail.com 
Keywords: active adaptation, multi-sensor system, distributed Kalman filtering, instrumental optimization performance criterion.

Received: $18^{\text {th }}$ May, 2019 / Revised: $19^{\text {th }}$ October, 2019 Accepted: $11^{\text {th }}$ November, $2019 /$ First online: $16^{\text {th }}$ December, 2019

Competing interests. We have no competing interests.

Authors' contributions and responsibilities. Each author has participated in the article concept development and in the manuscript writing. The authors are absolutely responsible for submitting the final manuscript in print. Each author has approved the final version of manuscript.

Funding. The reported study has been funded by the Russian Foundation for Basic Research and the Government of Ulyanovsk region according to the research projects nos. 18-41-732002 and 18-47-730001.

\section{References}

1. Katkovnik V. Ya., Poluektov R. A. Mnogomernye diskretnye sistemy upravleniia [Multidimensional Discrete Control Systems]. Moscow, Nauka, 1966, 416 pp. (In Russian)

2. Balakrishnan A. Kalman filtering theory. New York, Optimization Software, Inc., Publications Division, 1984, xii +222 pp.

3. Maybeck P. S. Stochastic Models, Estimation, and Control, vol.1, Mathematics in Science and Engineering, vol. 141. New York, Academic Press, Inc, 1979, xix +423 pp.

4. Fomin V. N. Rekurrentnoe otsenivanie $i$ adaptivnaia fil'tratsiia [Recurrent Estimation and Adaptive Filtering]. Moscow, Nauka, 1984, 288 pp. (In Russian)

5. Speyer J. Computation and transmission requirements for a decentralized linear-quadraticGaussian control problem, IEEE Trans. Automatic Control, 1979, vol. 24, no. 2, pp. 266-269. doi: 10.1109/TAC.1979.1101973.

6. Rao B. S., Durrant-Whyte H. F. Fully decentralised algorithm for multisensor Kalman filtering, IEE Proc.-Control Theory Appl., 1991, vol. 138, no. 5, pp. 413-420. doi: 10.1049/ ip-d.1991.0057.

7. Olfati-Saber R. Distributed Kalman filtering and sensor fusion in sensor networks, In: Networked Embedded Sensing and Control, Lecture Notes in Control and Information Science, 331; eds. P. J. Antsaklis, P. Tabuada. Springer, Berlin, Heidelberg, 2006, pp. 157-167. doi: $10.1007 / 11533382 \_10$.

8. Alriksson P., Rantzer A. Model based information fusion in sensor networks, IFAC Proceedings Volumes, 2008, vol.41, no. 2, pp. 4150-4155. doi: 10.3182/20080706-5-KR-1001. 00698.

9. Rao B. S. Y., Durrant-Whyte H. F., Sheen J. A. A fully decentralized multi-sensor system for tracking and surveillance, Int. J. Robot. Res., 1993, vol.12, no. 1, pp. 20-44. doi: 10. 1177/027836499301200102.

10. Mahmoud M. S., Khalid H. M. Distributed Kalman filtering: a bibliographic review, IET Control Theory and Applications, 2013, vol.7, no.4, pp. 483-501. doi:10.1049/iet-cta. 2012.0732.

11. Marelli D., Zamani M., Fu M., Ninness B. Distributed Kalman filter in a network of linear systems, Systems Control Letters, 2018, vol.116, no.6, pp. 71-77. doi:10.1016/j. sysconle.2018.04.005.

12. Wu Z., Fu M., Xu Yo., Lu R. A distributed Kalman filtering algorithm with fast finite-time convergence for sensor networks, Automatica, 2018, vol.95, no. 9, pp. 63-72. doi: 10.1016/ j. automatica.2018.05.012.

13. Dormann K., Noack B., Hanebeck U. D. Optimally distributed Kalman filtering with datadriven communication, Sensors, 2018, vol.18, no.4, pp. 1034. doi: 10.3390/s18041034. 
14. Badyn M. H., Mesbahi M. Large-scale distributed Kalman filtering via an optimization approach, IFAC Papers OnLine, 2017, vol. 50, no. 1, pp. 10742-10747. doi : 10.1016/j . ifacol. 2017.08 .2268$.

15. Govaers F. Distributed Kalman filter (Chapter 13), In: Kalman Filters - Theory for Advanced Applications; eds. Ginalber Luiz de Oliveira Serra. London, IntechOpen, 2018, pp. 253-272. doi: 10.5772/intechopen.71941.

16. Semushin I. V. The APA based time-variant system identification, In: 53rd IEEE Conference on Decision and Control (15-17 December 2014, Los Angeles, CA, USA), 2014, pp. 41374141. doi: 10.1109/CDC.2014.7040033.

17. Fletcher R. Practical Methods of Optimization. Chichester, Great Britain, John Wiley \& Sons Ltd, xiv+436 pp. doi: 10.1002/9781118723203.

18. Semushin I. V. Adaptation in stochastic dynamic systems - Survey and new results II, Int. J. Communications, Network, and System Sciences, 2011, vol.4, no.4, pp. 266-285. doi: 10.4236/ijens.2011.44032.

19. Tsyganova Yu. V. Othogonalized array methods for parametric identification of linear stochastic systems, Dissertation (Doct. Phys.-Math. Sci.; 05.13.18). Ulyanovsk, Ulyanovsk State University, 2017, 400 pp. (In Russian) 